九州大学学術情報リポジトリ

Kyushu University Institutional Repository

\title{
Measuring Inclination Angle of Semi-crawler Tractors Using MEMS Sensor
}

Choe, Jung Seob

Department of Precision Mechanical Engineering, Kyungpook National University

Mitsuoka, Muneshi

Department of Bioproduction Environmental Science, Graduate School of Kyushu University

Inoue, $\mathrm{E} \mathrm{i} \mathrm{j} \mathrm{i}$

Department of Bioproduction Environmental Science, Graduate School of Kyushu University

Okayasu, Takashi

Department of Bioproduction Environmental Science, Graduate School of Kyushu University

他

https://doi.org/10.5109/26164

出版情報：九州大学大学院農学研究院紀要. 58 (1)，pp.79-85，2013-02. Faculty of Agriculture， Kyushu University

バージョン :

権利関係 : 


\title{
Measuring Inclination Angle of Semi-crawler Tractors Using MEMS Sensor Jung Seob CHOE ${ }^{1}$, Muneshi MITUSOKA ${ }^{2 *}$, Eiji INOUE ${ }^{2}$, Takashi OKAYASU ${ }^{2}$, Yasumaru HIRAI ${ }^{2}$, Tae Wook KIM ${ }^{1}$, Soon Goo KWON ${ }^{3}$ and Jong Min PARK ${ }^{3}$
}

(Received October 31, 2012 and accepted November 8, 2012)

\begin{abstract}
In this study, we established a method to calculate inclination angle of moving vehicles from acceleration and angular velocity measured by microelectromechanical systems (MEMS) sensor, which is becoming popular recently due to its ultra-small size and low manufacturing cost. We applied the technique to measure inclination angle of semi-crawler tractor to validate its usefulness. Results from various methods to calculate inclination angle of a vehicle from acceleration and angular velocity data showed that moving average acceleration method and wavelet acceleration method produced results that significantly deviated from the actual inclination angle, while results from angular velocity method and acceleration-angular velocity method were close to the actual inclination angle. Inclination angle calculated by acceleration method in a static state was very similar to the measurement from inclination sensor and was highly accurate, but did not demonstrate sufficient accuracy in dynamic state as the estimated inclination angle was at maximum $10^{\circ}$ smaller at inclination angle of $75^{\circ}$. In the meanwhile, the inclination angle calculated by angular velocity method showed only small deviation from the sensor measurement even at dynamic state. As a result of measuring inclination of semi-crawler type tractor using MEMS sensor, it was determined that the angular velocity method is the most versatile of the four methods tested as it can not only produce very accurate time series of inclination angle but also be used to easily obtain angular displacement from the angular velocity data obtained. However, error of integration was shown to increase with longer measurement times and it must be addressed by an appropriate solution. Acceleration-angular velocity method also produced similar results as experiments were performed indoors where disturbance factors were few. The inclination angle measurement system using MEMS sensor established in this study can be applied to agricultural vehicles such as tractors as well as to almost all construction and general vehicles with drive system. We expect the system to contribute to development of a safety system that can prevent rollover accidents and quickly respond to them.
\end{abstract}

Key words: acceleration-angular velocity method, angular velocity method, inclination angle, MEMS sensor, microelectromechanical systems

\section{INTRODUCTION}

Of all agricultural accidents in Japan, deaths related to agricultural machinery account for about 300 cases each year and have not significantly decreased for the past 10 years despite continued effort from the government. About $45 \%$ of these deaths are caused by manned tractors, and of these about 70\% are fall and rollover accidents. Accurate data for relevant accidents are hard to find in Korea but it is expect to be similar as in Japan. These accidents are attributed to mistakes and carelessness on the part of the driver, and safety training is currently the only measure against such accidents.

However, such training in itself cannot be a fundamental solution to the problem. These accidents are caused not only by carelessness of the driver but also by other factors such as environmental factors and may occur inevitably. In order to prevent the accidents and reduce casualties, various solutions to this problem must be assessed (Rowe, E. P. H., 1976).

Department of Precision Mechanical Engineering, Kyungpook National University, Korea

2 Department of Bioproduction Environmental Science, Graduate School of Kyushu University, Japan

${ }^{3}$ Department of Bioindustrial Machinery Engineering, Pusan National University, Korea

* Corresponding author (E-mail: mitsuoka@bpes.kyushu-u. ac.jp)
As one of the possible solutions, research is currently being conducted in Korea and Japan to create a system in which the inclination sensors detect vehicle with an angle above a certain threshold to be in a rollover state and automatically send calls on the cell phone. Because many agricultural labors such as operation of tractor are performed by a single person, rescue mission after occurrence of an accident is often delayed. By automatically sending accident alert, the system being researched attempts to prevent serious injury or death due to ignorance of the accident. Also, the operator may be immersed in labor such that he or she is unaware of the risk of rollover; in this case, the system can detect the aberrant inclination angle and alert the operator to quickly reposition the vehicle, or automatically stop the vehicle to prevent accident.

The first step in establishing such system is to conveniently acquire operational data of the vehicle including its inclination angle.

Traditional method that measures vibration components of the vehicle in 6 directions ( 3 translational and 3 rotational directions) measured translational acceleration using translational accelerometer and rotational acceleration using gyro sensor. Also, a method is available for calculating 6-degrees-of-freedom vibration acceleration using multiple translational accelerometers (Inoue E. et al., 1990).

However, the abovementioned measurement systems 
are large and very expensive. Also, this system only measures the inclination angle of a vehicle and cannot monitor states of other operational parameters of the vehicle at work.

At the same time, agricultural support using information and communication technology and application of agricultural labor management system to enhance management efficiency are actively being researched, reflecting need for high-efficiency and utilization in agricultural machinery. Due to these changing trends, establishment of a measurement system that can monitor operational information of agricultural machinery including its safety has become increasingly important.

As a part of an effort to establish a measurement system that can comprehensively examine operational information of agricultural machinery, in this study, microelectromechanical systems (MEMS) sensor was used to measure acceleration and angular velocity of a moving object and a method for calculating inclination angle from these measurements was developed. By using MEMS sensor that is becoming popular recently due to its ultrasmall size and low manufacturing cost, we were able to significantly reduce measurement cost and also facilitated integration with additional system for acquiring operational information. We tested the system in measuring inclination angle of semi-crawler tractor in order to verify its usefulness.

\section{MATERIALS AND METHODS}

\section{Basic specifications of the tested MEMS sensor}

MEMS sensor integrates electrical circuit and other elements such as sensor and actuator on a silicon substrate, and can perform complicated functions while being small in size. Based on technique for manufacturing semiconductor integrated circuit, microfabrication technique that involves electrical, mechanical, photonic, and material technology is used to manufacture MEMS sensors. It is widely used as a high-value added fundamental component in various fields including information and communications, automobile, home appliances, industrial machinery, medicine and biology, and environment and disaster prevention.

Wireless 9-axis motion sensor

The MEMS sensor used in this study was manufactured by Logical Product, with capacity of 5G/300dps and is wireless 9-axis motion sensor that can receive and transmit data. It can wirelessly measure translational acceleration, rotational angular velocity, and geomagnetic orientation in 3 directions for each measure.

Conventional measurement system for acceleration and angular velocity are large and involved significant measurement costs. In contrast, the MEMS measurement sensor used in this study only costs $1 / 10$ of the conventional system, allowing for inexpensive measurement of acceleration and angular velocity.

This sensor is very light and compact with total weight of $35 \mathrm{~g}$ including the battery, can be easily installed, and only requires minimal installation space. It can also perform remote wireless measurement and data logging. Specified low power communication was selected for the private transceiver device used to transmit and receive command and to receive measurement data and file with the wireless sensor module, which allows wireless communication over about $50 \mathrm{~m}$.

Main specifications of the tested MEMS sensor are shown in Table 1.

Table 1. Main specifications of 9-axis wireless motion sensor

\begin{tabular}{ll}
\hline Modulation type & DS-SS \\
Radio frequency & $2405 \mathrm{MHz} 2480 \mathrm{MHz}, 5 \mathrm{MHz}$ interval \\
Battery & AAA battery $\times 1$ \\
Power consumption & Maximum $230 \mathrm{mV}$ \\
External dimensions & $40 \mathrm{~mm} \times 20 \mathrm{~mm} \times 55 \mathrm{~mm}$ \\
Weight & Approximately $35 \mathrm{~g}$ \\
\hline
\end{tabular}

By using this MEMS sensor, time series of acceleration and angular velocity in 3 directions for various vehicles including semi-crawler tractor can be continuously obtained. From the obtained data, inclination angle of the road can be easily calculated. Moreover, vehicle's operational status can be monitored from the acceleration and angular velocity data, which would enable low-cost establishment of a comprehensive control system for not only preventing rollover accidents but also managing and controlling general operation of the vehicle.

Acceleration sensor

Voltage supplied for acceleration sensor in the MEMS sensor is $3.3 \mathrm{~V}$ and output of the sensor at $0 \mathrm{G}$ is $1.65 \mathrm{~V}$. Sensor sensitivity is $190.0 \mathrm{mV} / \mathrm{g}$.

Output voltage of the acceleration sensor is $\mathrm{AD}$ converted into 12 bits. $\mathrm{AD}$ converted value is a straight binary, and full scale is $3.3 \mathrm{~V}$. Therefore, when the output voltage of the sensor is $\mathrm{V}, \mathrm{AD}$ converted value $\mathrm{X}_{\mathrm{acc}}$ is as in Eq. 1.

$$
\mathrm{X}_{\mathrm{acc}}=\frac{4095 \times \mathrm{V}}{3.3}
$$

When acceleration is $\mathrm{G}$, conversion equation is as in Eq. 2. Note that for actual output at $0 \mathrm{G}$, some offset is included due to factors such as temperature.

$$
G=\frac{\mathrm{V}-1.65}{0.19}
$$

Direction of each axis of the acceleration sensor is shown in Fig. 1.

Angular velocity sensor

Output from angular velocity sensor was filtered by a low pass filter with cutoff frequency of approximately $190 \mathrm{~Hz}$, amplified by 5.6 times using an op-amp, and converted by an $\mathrm{AD}$ converter.

The op-amp operates with reference to the reference voltage of gyro sensor $\left(V_{\text {ref }}=1.35 \mathrm{~V}\right)$, and difference between this reference voltage and sensor output is multiplied by 5.6 and entered into the AD converter. Sensor output at $0 \mathrm{deg} / \mathrm{sec}$ (deg/sec is hereafter referred to as $\mathrm{dps}$ ) is $1.35 \mathrm{~V}$. Sensor sensitivity is $0.67 \mathrm{mV} / \mathrm{dps}$.

When output voltage of the sensor is $\mathrm{V}, \mathrm{AD}$-converted value $\mathrm{X}_{\text {gyro }}$ is as in Eq. 3 . 


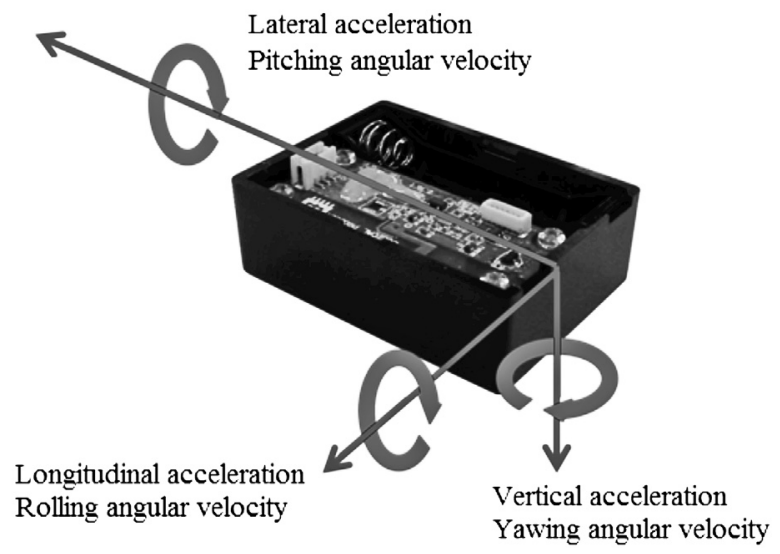

Fig. 1. Direction of each axis in MEMS sensor.

$$
X_{\text {gyro }}=\frac{4095 \times \mathrm{V}}{3.3}
$$

When angular velocity is $\mathrm{W}$, conversion is given by Eq. 4.

$$
W=\frac{V-1.35}{5.6 \times 0.00067}
$$

Direction of each axis in angular velocity sensor is shown in Fig. 1.

\section{Accuracy of the tested MEMS sensor Calibration}

Because MEMS acceleration sensors have some variability across sensors, it should be calibrated to give gravitational acceleration of $1 \mathrm{G}$ and $-1 \mathrm{G}$ when the sensor is right-side up and upside down, respectively. In this study, calibration was performed in the form of a linear function for all acceleration data, as in Eq. 5 .

$$
y=\mathrm{a} x+\mathrm{b}
$$

Here, $\mathrm{x}$ and $\mathrm{y}$ are respectively the data before and after calibration, a and $\mathrm{b}$ are correction factors whose values are given in Table 2 .

Table 2. Correction factors for acceleration sensor

\begin{tabular}{cccc}
\hline & $\begin{array}{c}\text { Acceleration X } \\
\text { (Longitudinal } \\
\text { acceleration) }\end{array}$ & $\begin{array}{c}\text { Acceleration Y } \\
\text { (Lateral } \\
\text { acceleration) }\end{array}$ & $\begin{array}{c}\text { Acceleration Z } \\
\text { (Vertical } \\
\text { acceleration) }\end{array}$ \\
\hline $\mathrm{a}$ & 9.6060 & 9.6500 & 9.8984 \\
$\mathrm{~b}$ & 0.0752 & -0.1957 & -1.4436 \\
\hline
\end{tabular}

\section{Inclination sensor}

In order to verify reliability of the inclination angle measured by MEMS sensor, a commercially available inclination sensor was used to measure the inclination angle. Inclination angles measured by the two sensors were then compared.

The inclination sensor used for the experiment was VTI Technology's SCA121T-D03 for XY dual-axis inclination measurement, and has analog voltage output, high accuracy, outstanding shock resistance and durability, and long-term stability.

Table 3 shows main specifications of the inclination sensor.

Table 3. Specifications of the inclination sensor SCA121T-D03

\begin{tabular}{ll}
\hline Measurement range & $\pm 90^{\circ}$ \\
Input voltage & $7 \sim 35 \mathrm{~V}$ \\
Offset (output when horizontal) & 2.5 \\
Sensitivity & $2 \mathrm{~V} /$ \\
Response frequency & $8 \sim 28 \mathrm{~Hz}$ \\
\hline
\end{tabular}

\section{Static experiment}

Dynamic and static experiments were performed to verify accuracy of the MEMS sensor.

For static experiment, level meter, inclination sensor, and MEMS sensors were fixed on an acrylic plate and measured angle at 0,30 , and $60^{\circ}$ for 10 seconds each.

Acceleration data in 3 directions for the MEMS sensor was corrected according to calibration, and averages were taken for each of X, Y, and Z axis. From these values, acceleration method was used to calculate inclination angle and compared to the value measured from the inclination sensor, as shown in Table 4.

From these results, accuracy of the MEMS sensor was shown to be comparable to the inclination sensor.

Table 4. Inclination angle measurement at static state

\begin{tabular}{llll}
\hline Level meter & $0^{\circ}$ & $30^{\circ}$ & $60^{\circ}$ \\
\hline Inclination sensor & 0.00000025 & 29.78 & 60.95 \\
MEMS sensor & 0.31 & 29.85 & 60.79 \\
\hline
\end{tabular}

\section{Dynamic experiment}

Experiment for characterizing dynamic accuracy of the MEMS sensor was performed using two methods.

First, acrylic plate with inclination sensor and MEMS sensor attached to it was gradually tilted from the horizontal state over 40 seconds then slowly reverted back to the horizontal state. Next, acrylic plate was shaken to change its angle relatively quickly before being positioned back at the horizontal level.

Results of this experiment will be detailed in the results and discussion section.

\section{Measurement of inclination angle of semi-crawler tractor}

A four-wheel drive, 15 horsepower Kubota GB150-PC semi-crawler tractor in Fig. 2 was used for the experiment. For measurement of acceleration and angular velocity, MEMS sensor was installed in the driver's seat.

Measurement of inclination angle of tractor in operation was performed while tractor passed through a perpendicular triangle-shaped inclined plane with base length of $122.58 \mathrm{~cm}$ and height of $28.82 \mathrm{~cm}$ on even, dry, and rigid road. In order to maintain vibration state as constant as possible during drive, the tractor was unmanned and moved forward without any manipulation of the 


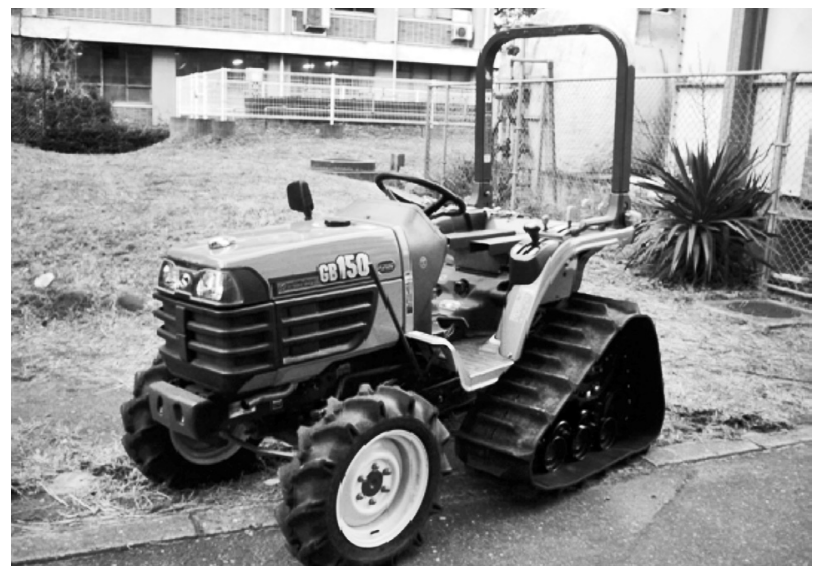

Fig. 2. Tractor tested in the study.

drive wheel. Drive speeds for the experiment were $0.25 \mathrm{~m} / \mathrm{s}$ and $0.5 \mathrm{~m} / \mathrm{s}$, and for each speed measurement was taken 6 times for going uphill and downhill respectively, with total of 24 measurements. Of 6 measurements, the vehicle was stopped in middle of the inclined plane for the first 3 measurements and the vehicle completely passed through the inclined plane to return to horizontal state for the last 3 measurements.

\section{Methods for calculating inclination angle}

We tested 3 methods for obtaining inclination angle of the road from data transmitted by the MEMS sensor. Explanation for each method is given below(Roberto N. Barbosa et al., 2007).

\section{Acceleration method}

$\mathrm{X}$-axis and $\mathrm{Z}$-axis components of the acceleration data from the MEMS sensor was calibrated and removed of high-frequency components. Time series of inclination angle was calculated using Eq. 6.

$$
\theta=\arctan \left(\frac{\alpha_{x}}{\alpha_{z}}\right) \times\left(\frac{180}{\pi}\right)
$$

\section{Angular velocity method}

Y-axis component of angular velocity data of the MEMS sensor was calibrated, and Eq. 7 was used for integration using mensuration of division to calculate time series of angular displacement in the Y-axis. This data was used to obtain time series of inclination angle.

$$
\theta_{k+1}=\left(\omega_{y k}+\omega_{y k+1}\right) \times \Delta t \div 2+\theta_{k}
$$

\section{Acceleration-angular velocity method}

Acceleration output signal from the MEMS sensor was low-pass filtered, and integrated output signal of angular velocity was high-pass filtered. Then Eq. 8 was used to obtain time series of inclination angle.

$$
\theta_{k+1}=\theta_{k}-\phi \theta_{k} \Delta t+\phi \alpha \Delta t+\omega \Delta t
$$

Here, $\phi=1 / T, T=2 \pi f_{0}$, and $f_{0}$ is cut-off frequency.

\section{RESULTS AND DISCUSSION}

\section{Accuracy of the MEMS sensor}

As mentioned in the previous section, accuracy of the MEMS sensor at static state was very high and almost as accurate as the inclination sensor.

Inclination angle calculated by the acceleration method from the measurement of gradual angle change at dynamic state was compared to the inclination angle measured by the inclination sensor and shown in Fig. 3. As shown in the figure, the acceleration method underestimated the inclination angle by $10^{\circ}$ at maximum compared to the measurement by the inclination sensor. The error was attributed to noise in the measurement signal from various sources. In order to apply the acceleration method in actual applications, subsequent research to block to remove this noise would be required.

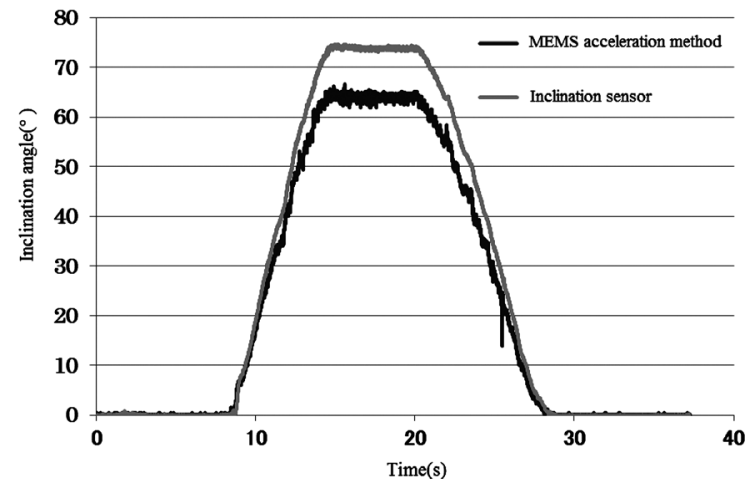

Fig. 3. Comparison between inclination angle estimated by the acceleration method and inclination angle measured by the inclination sensor with gradual angle changes.

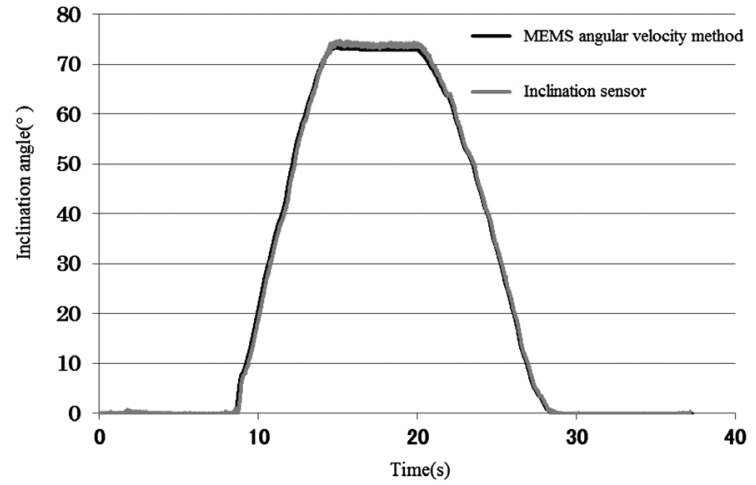

Fig. 4. Comparison between inclination angle estimated by the angular velocity method and inclination angle measured by the inclination sensor with gradual angle changes.

Fig. 4 compares inclination angle calculated by the angular velocity method from the measurement of gradual angle changes at dynamic state was compared to the inclination angle measured by the inclination sensor. As shown in the figure, the two inclination angles were very similar. Therefore, calculation of inclination angle of MEMS sensor using angular velocity is sufficiently accurate to be used for assessing inclination in various vehicles. 
In addition to these techniques, moving average acceleration method and wavelet acceleration method were used for calculation of inclination angle, but error was significantly large for these two methods. For measurement with vibration added, only results for angular velocity method are shown as in Fig. 5.

In Fig. 5, inclination angle measured by MEMS sensor under considerable vibration condition deviated slightly from the measurement from the inclination sensor but there was effectively no time delay.

From the above results, MEMS sensor was demonstrated to be capable of accurately measuring inclination angle under both gradual angle changes and rapid angle changes accompanied by vibration.

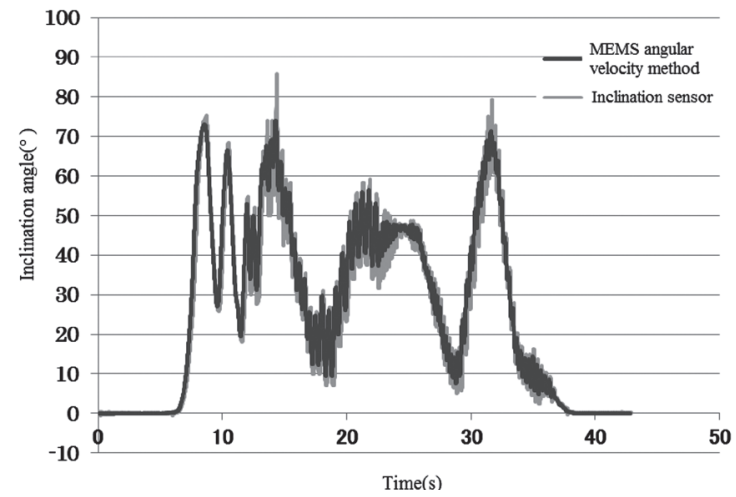

Fig. 5. Comparison of inclination angle measured by the angular velocity method and inclination angle measured by the inclination sensor under irregular angle changing state with vibration.

\section{Measurement of inclination angle of tractor}

As mentioned in the Materials and methods section and in the Accuracy of the MEMS sensor part of this section, preliminary study showed that the moving average acceleration method and the wavelet acceleration method did not produce precise inclination angle over time due to vibration of the incline and resulted in significantly deviating results as it incorporated acceleration during starting and stopping of the vehicle into the inclination angle. Therefore, results from only the angular velocity method and the acceleration-angular velocity method are presented in this section. Note that $\mathrm{f}_{\mathrm{o}}=0.005$ for the acceleration-angular velocity method.

Figs. 6 through 13 show results of inclination angle calculated from the angular velocity method and the acceleration-angular velocity method using measurements from each of the following conditions using the method detailed in the Materials and methods section: drive speeds of $0.25 \mathrm{~m} / \mathrm{s}$ and $0.5 \mathrm{~m} / \mathrm{s}$, going uphill or downhill and then stopping, and going uphill or downhill through the inclined plane.

The angular velocity method was evaluated to be a highly versatile technique because it can estimate time series of inclination angle with high precision and yield angular displacement from the angular velocity measurement. However, error of integration increases with longer measurement time and appropriate solution would be needed.
In this experiment, the acceleration-angular velocity method did not significantly differ in performance compared to the angular velocity method. However, this was determined to be due to experiments being performed at a site with relatively few disturbance factors.

Apart from somewhat destabilizing trend at higher speeds, difference due to speed was not observed.

A slight error in measurement was observed when vehicle passed through the inclined plane and returned to the horizontal state for both uphill and downhill conditions. This was attributed to the integration error from lengthened measurement time as mentioned earlier. This trend was larger in the acceleration-angular velocity method compared to the angular velocity method.

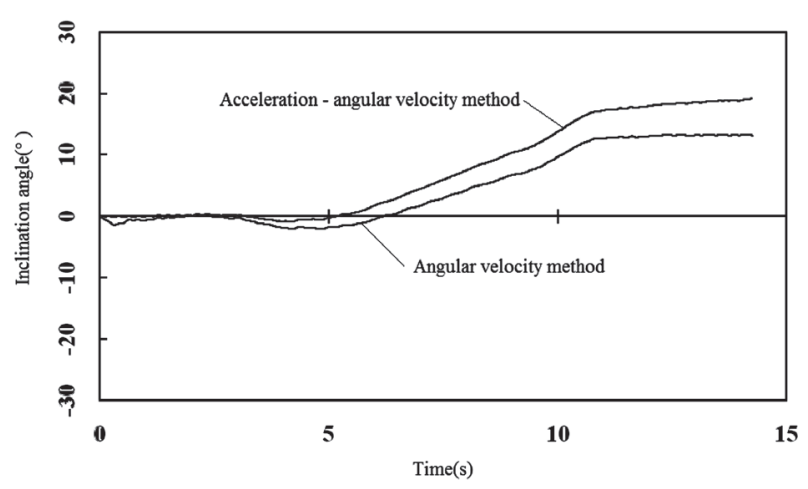

Fig. 6. Vehicle's inclination angle when going uphill at $0.25 \mathrm{~m} / \mathrm{s}$ before stopping.

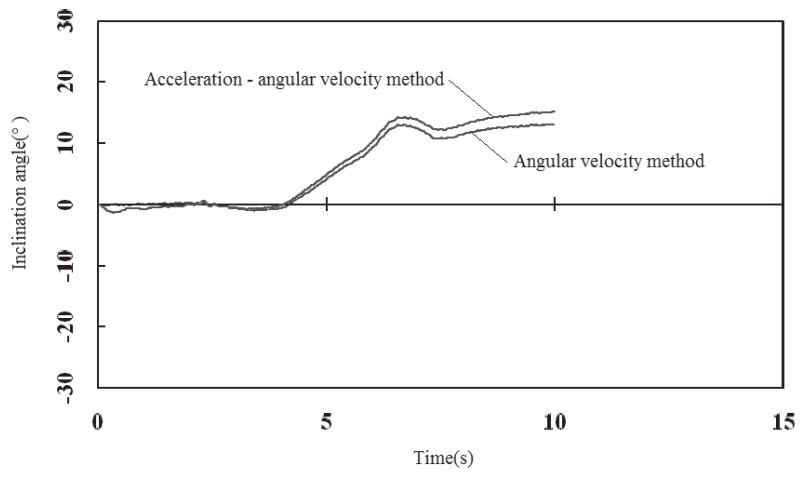

Fig. 7. Vehicle's inclination angle when going uphill at $0.5 \mathrm{~m} / \mathrm{s}$ before stopping.

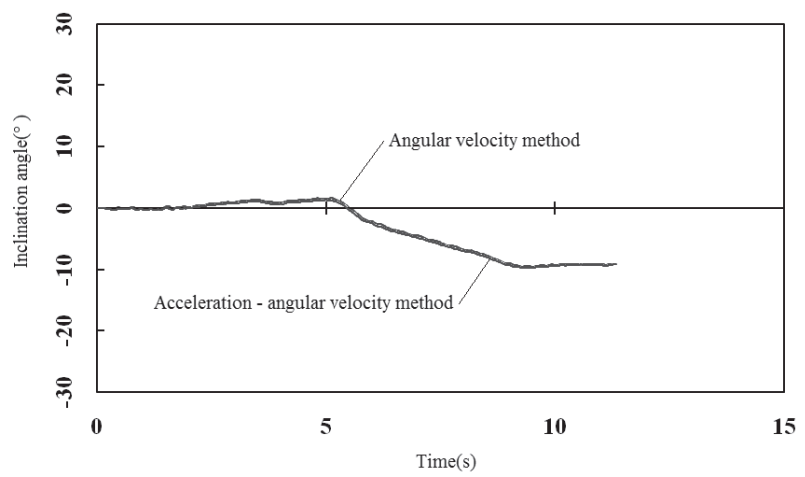

Fig. 8. Vehicle's inclination angle when going downhill at $0.25 \mathrm{~m} / \mathrm{s}$ before stopping. 


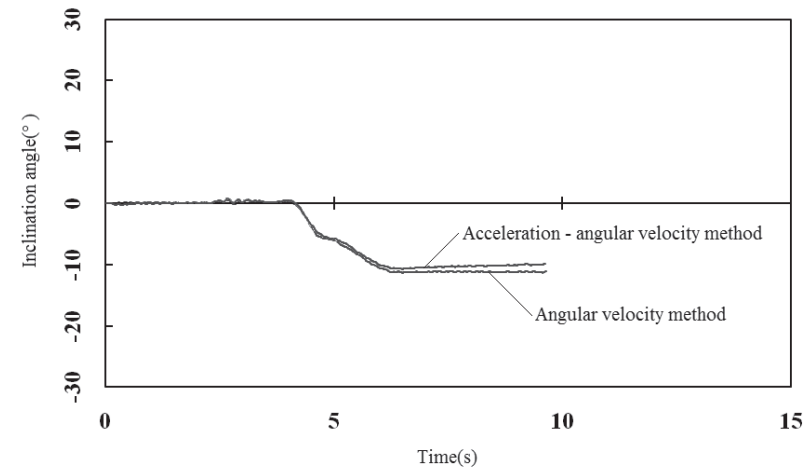

Fig. 9. Vehicle's inclination angle when going downhill at $0.5 \mathrm{~m} / \mathrm{s}$ before stopping.

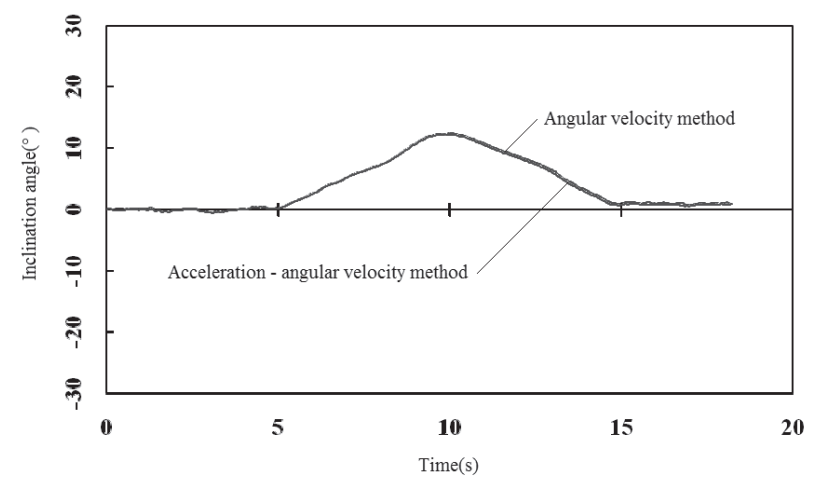

Fig. 10. Vehicle's inclination angle when going uphill at $0.25 \mathrm{~m} / \mathrm{s}$ through the inclined plane.

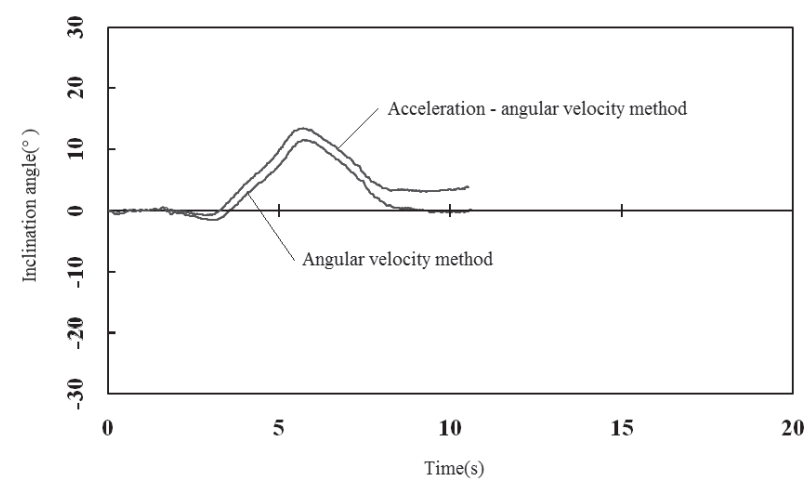

Fig. 11. Vehicle's inclination angle when going uphill at $0.5 \mathrm{~m} / \mathrm{s}$ through the inclined plane.

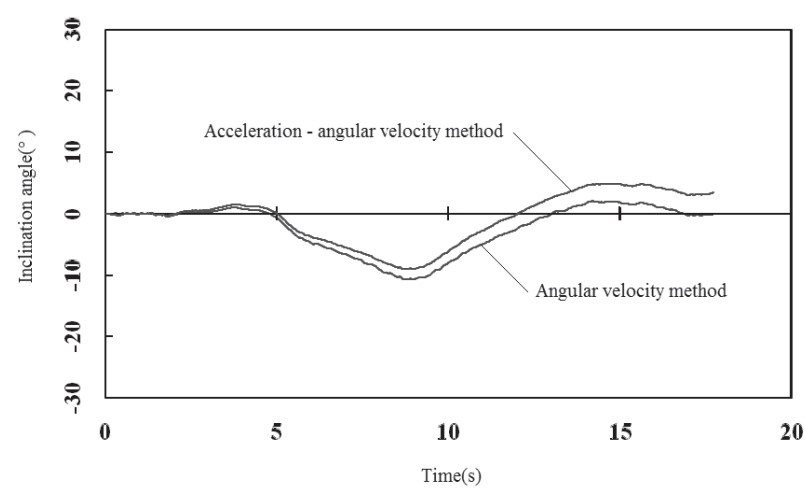

Fig. 12. Vehicle's inclination angle when going downhill at $0.25 \mathrm{~m} / \mathrm{s}$ through the inclined plane.

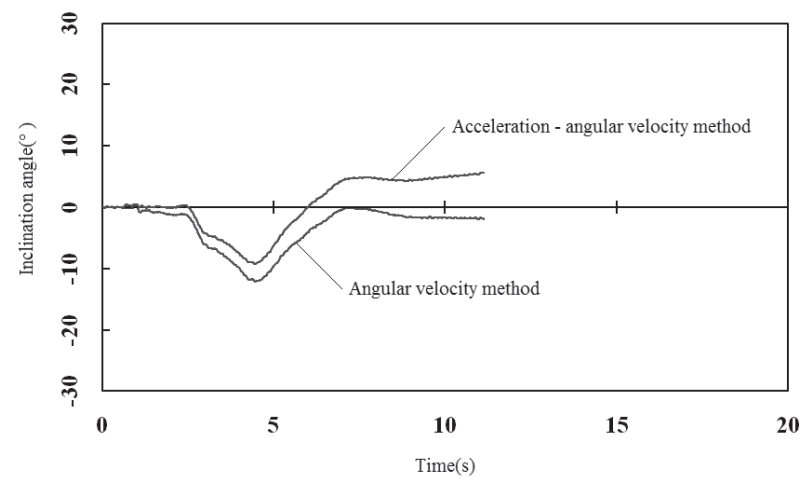

Fig. 13. Vehicle's inclination angle when going downhill at $0.5 \mathrm{~m} / \mathrm{s}$ through the inclined plane.

\section{SUMMARY AND CONCLUSION}

As a part of research for establishing measurement system that can comprehensively monitor operational information of agricultural machinery, we used smallsized and low-cost MEMS sensor to measure acceleration and angular velocity of moving bodies and calculate inclination angle from these measurements. This method was applied to measurement of inclination angle in an actual semi-crawler tractor to verify its usefulness. Main findings are as follows.

1. Using acceleration and angular velocity measured by MEMS sensor to calculate inclination angle of vehicle was evaluated. Calculations of inclination angle by the moving average acceleration method, wavelet acceleration method, angular velocity method, and acceleration-angular velocity method showed that results from the moving average acceleration method and wavelet acceleration method significantly deviated from the actual inclination angle of the vehicle while measurements from the angular velocity method and the acceleration-angular velocity method were similar to the actual angle.

2. Inclination angle calculated by the acceleration method from the MEMS sensor measurements at static state was almost identical to the measurement by the inclination angle and displayed high accuracy.

3. Inclination angle calculated by the acceleration method from the measurement at dynamic state with gradual angle changes was smaller than the value measured by the inclination sensor by over $10^{\circ}$ at $75^{\circ}$ and was not sufficiently accurate. However, inclination angle calculated by the angular velocity method almost coincided with the measurement by the inclination sensor.

4. As a result of measuring inclination angle of semicrawler type tractor using MEMS sensor, the angular velocity method can not only accurately produce time series of inclination angle but also facilitate measurement of angular displacement from the angular velocity data. However, integration error increased with longer measurement time and appropriate solution would be required. Because experiments were performed indoors where disturbance factors are mini- 
mal, the acceleration-angular velocity method performed comparable to the angular velocity method.

The inclination angle measurement system using MEMS sensor established in this study can be applied to agricultural vehicles such as tractors as well as to almost all construction and general vehicles with drive system. We expect the system to contribute to development of a safety system that can prevent rollover accidents and quickly respond to them.

\section{ACKNOWLEDGEMENTS}

This Research was supported by Kyungpook National University Research Fund, 2011.

\section{REFERENCES}

Inoue E., J. Sakai and S. Inaba 1990 Basic Studies on Vibration Characteristics of the Rubber Crawler System for Farm
Machinery (Part 1), Journal of the Japanese Society of Agricultural Machinery, 52(1): 27-34

Inoue E., J. Sakai and S. Inaba 1990 Basic Studies on Vibration Characteristics of the Rubber Crawler System for Farm Machinery (Part 2), Journal of the Japanese Society of Agricultural Machinery, 52(4): 29-36

Inoue E., J. Sakai and S. Inaba 1990 Basic Studies on Vibration Characteristics of the Rubber Crawler System for Farm Machinery (Part 3), Journal of the Japanese Society of Agricultural Machinery, 52(5): 11-18

Inoue E., J. Sakai and S. Inaba 1990 Basic Studies on Vibration Characteristics of the Rubber Crawler System for Farm Machinery (Part 4), Journal of the Japanese Society of Agricultural Machinery, 52(6): 19-26

Roberto N. Barbosa, B. Wilkerson John, C. Yoder Daniel and H. Paul Denton 2007 Different sensing techniques for geomorphometric measurements, Computers and Electronics in Agriculture, 59: 13-20

Rowe, E. P. H. and H. B. Spencfr 1976 An Instrumented Tractor for Use in Motion Behavior Studies on Sloping Ground. I. Agric. Engng Res, 21: 355-360 